\title{
Effectiveness of SaeboReJoyce in the evaluation of the improve- ment of the occupational performance in Parkinson's disease: an outcome research
}

\author{
Salviani Silvia ${ }^{[1]}$, Tofani Marco ${ }^{[2]}$, Fabbrini Giovanni ${ }^{[3]}$, Leo Antonio ${ }^{[4]}$, Berardi An- \\ $\mathrm{na}^{[1]}$, Sansoni Julita ${ }^{[5]}$, and Galeoto Giovanni ${ }^{[5]}$ \\ ${ }^{1}$ Sapienza Università di Roma, Rome, Italy \\ ${ }^{2}$ Neurorehabilitation Unit, Bambino Gesù Children's Hospital, Rome, Italy \\ ${ }^{3}$ Department of Human Neurosciences Sapienza University of Rome and IRCCS Neuromed \\ Pozzili, Italy \\ ${ }^{4}$ Officine Ortopediche Rizzoli Srl, Rome, Italy \\ ${ }^{5}$ Department of Public Health and Infectious Diseases, Sapienza University of Rome, Rome, \\ Italy \\ giovanni.galeoto@uniromal.it
}

\begin{abstract}
The aim of this study was to assess the effectiveness of SaeboReJoyce in the evaluation of improvement of the occupational performance, in Parkinson's disease. Six patients with Parkinson's disease were recruited between June 2018 and September 2018 from the Parkinson's disease Outpatient Clinic of "Policlinico Umberto I" hospital in Rome. We assessed the effectiveness of SaeboReJoyce in evaluating the improvement of the occupational performance in Activities of Daily Living and Instrumental Activities of Daily Living. We chose four different activities that patients performed for five weeks with two weekly sessions. The impact on the quality of life, movements, fine and gross grasps, depression and satisfaction were evaluated. The analysis of the data from the rating scales and the tests shows an improvement in the functionality of the upper limb. The results for the rating scales are not statically significant but clinically relevant, while results for the ReJoyce are in part statistically significant. Our results suggest that the SaeboReJoyce can be a useful instrument not only for the health professional dealing with Parkinson Disease but also for nursing and rehabilitation sciences students for the evaluation of changes in functionality of the upper limb, including both gross and fine motor tasks/grasps, for patients with Parkinson Disease.
\end{abstract}

Keywords: SaeboReJoyce, ADL, IADL, Parkinson's Disease, Upper Limb.

\section{Introduction}

Parkinson's disease (PD) is a common progressive, neurodegenerative disease, affecting $0.1 \%$ of the general population and $1 \%$ of the population over 65 years [1]. In 2016, the estimated regional incidence rate of PD in Italy was 0.28 new cases/1000 person-years, with a prevalence of 3.89/1000 persons [2]. The Global Burden of Disease Study estimates that by 2040 as many as 12.9 million individuals will be affected, due to the aging of the world's population, and keeping in consideration that the incidence of PD increases with age [3].

PD has relevant clinical, social and economic implications. Disability in PD is due to the presence of both motor and non-motor symptoms, which restrict both the self- 
sufficiency and social participation of patients, leading to a low quality of life (QoL) for both patients and their caregivers [4].

The rating scales and standardized treatments currently available in Occupational Therapy are insufficient, thus leading to a difficulty in both students training and drafting a correct intervention strategy for the patients.

The SaeboReJoyce [5] is a rehabilitation device that allows to measure and evaluate the range of movement of the upper limb and different types of grasps. It has been used in tetraplegic patients jointly with the functional electrical stimulation and the conventional exercise therapy [6,7]. The SaeboReJoyce offers an additional tool to face this problem, being a novel rehabilitation device designed to measure, evaluate and increase the strength of muscles and the range of motion of joints of the upper extremities [5].

To date, this tool has been used with $\mathrm{C} 5-\mathrm{C} 7$ tetraplegic patients to play computer games associated with Activities of Daily Living (ADLs) [6,7].

The aim of this study was to evaluate its effectiveness in the evaluation of the improvement of occupational performance and functionality of the upper limb in ADLs and Instrumental Activity of Daily Living (IADLs), in PD.

\section{Material and Methods}

\subsection{Population}

The present study was conducted by health professionals of Sapienza University of Rome and ROMA - Rehabilitation \& Outcome Measures Assessment Association.

We included patients from the Parkinson's disease Outpatient clinic of "Policlinico Umberto I" hospital in Rome from June 2018 to September 2018. The inclusion criteria were diagnosis of Parkinson's disease, age $>40$ years, Montreal Cognitive Assessment MoCA [8] score $\geq 26$ and knowledge of the Italian language. For detecting mild cognitive impairment during patients' selection, we used the MoCA, a 30-point test administered in 10 minutes (cut off'26) [8].

\subsection{Rating scale and tests}

To evaluate QoL we used a patient-assessed instrument, the Parkinson's Disease Questionnaire-39 (PDQ-39) the most thoroughly tested and applied assessment tool in PD patients. It consists of 39 items, divided into 8 subtests: mobility, activities of daily living, emotional wellbeing, stigma, social support, cognition, communication and bodily discomfort ( $0=$ not disability; $156=$ severe disability) [9]. We use the Disability of the Arm, Shoulder and Hand (DASH) to identify symptoms and functions of the upper limb in patients with neurological or orthopedic disorders and it consists of 30 questions ( $0=$ not disability; 100=severe disability) [10]. The Jebsen-Taylor Hand Function Test (JTHFT) is a standardized test to evaluate the upper limb functionality through 7 items that simulated ADL [11]- The ReJoyce 
Arm-Hand Function Test (RAHFT) reproduces every movement and gross and fine motor tasks/grasps possible with the SaeboReJoyce [4, 5]. We performed three evaluations, during the first $\left(\mathrm{T}_{0}\right)$, the fifth $\left(\mathrm{T}_{1}\right)$ and the last $\left(\mathrm{T}_{2}\right)$ session. At last, we used the Geriatric Depression Scale (GDS) to evaluate the level of depressive symptoms over the past week considers the patient's point of view. It is useful for the screening and measurement of the degree of perceived depression in patients with PD and it is composed by 30 items $[12,13]$.

\subsection{Follow-up evaluation}

As a preliminary step we carried out an interview with each participant during which every person was specifically informed about the procedures and aims of the study; we collected personal data necessary for the research after the acquisition of informed consent from each participant. The evaluations were performed using selfadministrated measurement tools during the first $\left(T_{0}\right)$ and the last $\left(T_{1}\right)$ session. Only the ReJoyce Arm-Hand Function Test was performed three times, during the first $\left(\mathrm{T}_{0}\right)$, the fifth $\left(\mathrm{T}_{1}\right)$ and the last $\left(\mathrm{T}_{2}\right)$ session.

\subsection{Intervention}

The treatment lasted five weeks with two weekly sessions lasting about 45 minutes, except the first and the last meeting. In fact, during the first session, we outlined the project, obtained informed consent and in both the first and last sessions. The evaluations were performed using the measurement tools.

The SaeboReJoyce System is made up of 4 parts: the base, that clamps to a desk; the arm supports the manipulandum and provides resistance to motion in all directions; the manipulandum that consists of the peg, coins, jar lid, doorknob, key, gripper and handles used to play the computer games; and the PC Laptop that runs the interactive SaeboReJoyce NeuroGaming ${ }^{\mathrm{TM}}$ software.

The 4 chosen activities included: Bartender, Catcher, Weedo and Stack Attack. The goal of Barthender is to renovate the bar by pouring beverages for patrons based on their orders as fast and accurately as possible. The player has to select the beverage moving the manipulandum to the top of the screen over the requested bottle and grasp it. Once come back to the counter-top, a glass will appear and the player must "swing the gripper to pour until the golden marker". The goal of Catcher is to catch as many of the falling color-coded objects in the color-coded buckets as possible. We chose the jar lid like control. The player of Weedo must protect the flowers by removing weeds and dropping them into the compost heaps situated on either side of the screen. Like control, we chose the peg. The player of Stack Attack must eliminate falling blocks as they pass through bumpersby performing the displayed hand function. The game is over if too many blocks stack at the bottom of the screen. The preset hand functions are: gripper, peg, doorknob and jar lid in either directions [5]. The 4 chosen activities are illustrated in Figure 1 a,b,c,d. 


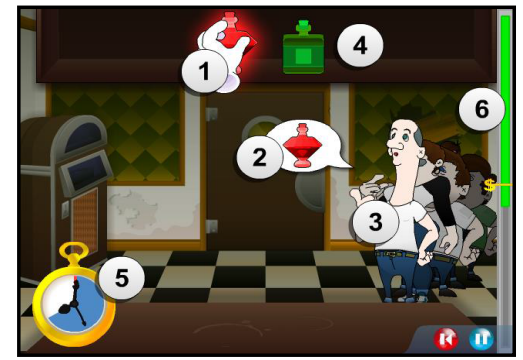

Figurela- The interactive scenarios Bartender.

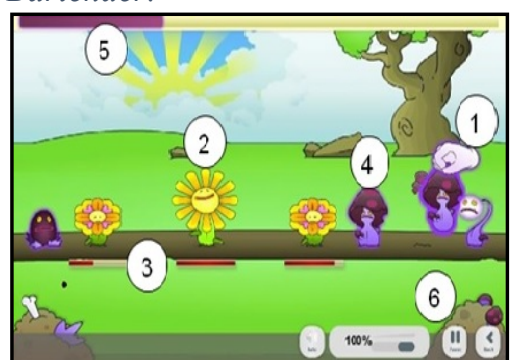

Figure1c The interactive scenarios Wedoo

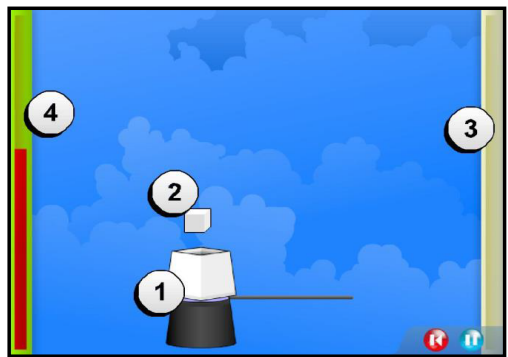

Figure1b- The interactive scenarios of Catcher.

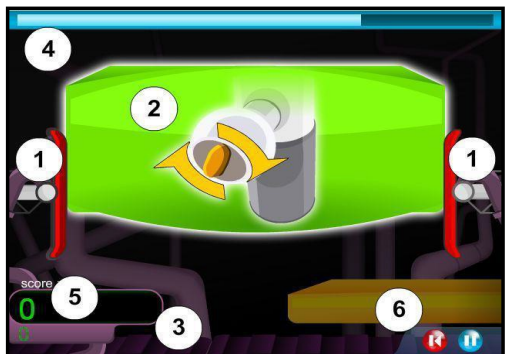

Figure1d- the interactive scenarios of Stack Attack.

\subsection{Analysis of data}

We used the descriptive analysis of general information (age, sex, diagnosis, year of diagnosis, Hoehn\&Yahr stage, education, employment, marital status).

Concerning the SaeboReJoyce test results, we grouped data in 3 groups, by movement (left, right, up, down, forwards and backwards), rotational movement (doorknob, key and jar) and gross and fine grasps (grip, peg and coin). For each group, the data were analysed both in terms of time required to complete the action (time) and quality of execution (value).

In order to provide a reliable evaluation of the change in upper limb functionality, we used the non-parametric Wilcoxon test (Z). The significance level has been set for $\mathrm{p}$-value less than or equal to 0.05 . The statistical analysis was performed through the Statistical Package of Social Science (SPSS) - version 23.0 for Windows.

\section{Results}

Fourteen patients were recruited for this study between June 2018 and September 2018. They all signed the written informed consent $[15,16]$ and six patients completed the study. Mean age of participants was $65.38 \pm 5.26 \mathrm{SD}$ and the mean MoCA score was $26.75 \pm 0.89$ SD. The features of the sample are presented in Errore. L'origine 
riferimento non è stata trovata. 1 . The results of the rating scales and of the Jebsen test are reported respectively in Table $\mathbf{2}$ and $\mathbf{3}$. The statistically significant data of the SaeboReJoyce test are summarized in Table 4.

Table 1. Features of the 6 participants.

\begin{tabular}{|l|l|}
\hline & Sample $=6$ \\
\hline Gender Male N (\%) & $5(83,3)$ \\
\hline Age Mean (SD) & $65.50 \pm 5.92$ \\
\hline Years from diagnosis Mean (SD) & $6.33 \pm 5.39$ \\
\hline MoCA Mean (SD) & $26.83 \pm 0.98$ \\
\hline H\&Y N (\%) & $1(16.7)$ \\
\hline 1 & $2(33.3)$ \\
\hline 2 & $3(50)$ \\
\hline 3 & $2(33.3)$ \\
\hline Education N (\%) & $1(16.7)$ \\
\hline Elementary School & $3(50)$ \\
\hline Middle School & $2(33.3)$ \\
\hline High School & $1(16.7)$ \\
\hline Employment N (\%) & $3(50)$ \\
\hline Employed & $5(62.5)$ \\
\hline Unemployed & $1(16.7)$ \\
\hline Retired
\end{tabular}

Table 2. Z-test results of the rating scales PDQ-39, DASH and GDS.

\begin{tabular}{ccccc}
\hline & $\mathrm{T}_{0} \boldsymbol{\mu} \pm$ SD & $\mathrm{T}_{1} \boldsymbol{\mu} \pm$ SD & Test & \\
\hline & & & $\mathrm{Z}$ & $\mathrm{p}$ \\
PDQ-39 & $84.00 \pm 32.00$ & $77.00 \pm 26.28$ & -.674 & 0.500 \\
DASH & $62.88 \pm 32.63$ & $55.33 \pm 22.18$ & -.530 & 0.596 \\
GDS & $11.63 \pm 6.30$ & $10.33 \pm 8.14$ & -.135 & 0.892 \\
\hline
\end{tabular}

Table 3. Z-test results of the Jebsen test; $D H=$ dominant hand; $N D H=$ non-dominant hand.

\begin{tabular}{ccccc}
\hline & $\mathrm{T}_{0} \boldsymbol{\mu} \pm$ SD & $\mathrm{T}_{1} \boldsymbol{\mu} \pm \mathbf{S D}$ & Test $\mathbf{Z}$ & $\mathbf{p}$ \\
\hline Item 1 DH & $48.00 \pm 56.80$ & $27.38 \pm 22.84$ & -.734 & 0.463 \\
Item 2 DH & $12.47 \pm 9.77$ & $7.14 \pm 3.67$ & -.943 & 0.345 \\
Item 3 DH & $12.22 \pm 8.85$ & $8.14 \pm 2.17$ & -.943 & 0.345 \\
Item 4 DH & $31.95 \pm 59.88$ & $8.65 \pm 2.37$ & -1.782 & 0.075 \\
Item 5 DH & $10.44 \pm 4.57$ & $8.33 \pm 4.49$ & -1.363 & 0.173 \\
\hline
\end{tabular}




\begin{tabular}{ccccc}
\hline Item 6 DH & $10.56 \pm 11.49$ & $5.67 \pm 1.87$ & -.674 & 0.500 \\
Item 7 DH & $27.71 \pm 61.55$ & $5.57 \pm 2.18$ & -.105 & 0.917 \\
Item 1 NDH & $68.12 \pm 29.02$ & $60.36 \pm 22.93$ & -.734 & 0.463 \\
Item 2 NDH & $9.66 \pm 2.72$ & $7.84 \pm 2.55$ & -1.363 & 0.173 \\
Item 3 NDH & $9.76 \pm 2.26$ & $9.63 \pm 2.25$ & -.105 & 0.917 \\
Item 4 NDH & $13.85 \pm 3.14$ & $12.48 \pm 3.78$ & -.105 & 0.917 \\
Item 5 NDH & $10.10 \pm 3.78$ & $8.62 \pm 3.16$ & -1.572 & 0.116 \\
Item 6 NDH & $7.21 \pm 2.37$ & $6.41 \pm 2.28$ & -.524 & 0.600 \\
Item 7 NDH & $6.86 \pm 2.10$ & $5.92 \pm 2.15$ & -.314 & 0.753 \\
\hline
\end{tabular}

Table 4. Z-test results of the ReJoyce test.

\begin{tabular}{|c|c|c|c|c|c|}
\hline & $\mathrm{T}_{0} \boldsymbol{\mu}$ (SD) & $\mathrm{T}_{1} \boldsymbol{\mu}(\mathbf{S D})$ & $\mathrm{T}_{2} \boldsymbol{\mu}(\mathbf{S D})$ & $\begin{array}{l}T_{0}-T_{1} \\
p \text { value }\end{array}$ & $\begin{array}{l}T_{0}-T_{2} \\
p \text { value }\end{array}$ \\
\hline Movement to left - RH - value & $64(25.5)$ & $93.3(11.5)$ & $98.6(2.2)$ & $0.043^{*}$ & $0.043 *$ \\
\hline Movement to right - $\mathrm{RH}$ - value & $57.4(25.2)$ & $82.8(22.1)$ & $86(12.8)$ & 0.068 & $0.043^{*}$ \\
\hline $\begin{array}{l}\text { Gross grasp - movement - release - } \\
\text { RH - value }\end{array}$ & $61(27.3)$ & $88.7(3.7)$ & $90.7(2.9)$ & $0.028^{*}$ & $0.027 *$ \\
\hline Gross grasp - release - RH - value & $81.7(25.75)$ & $95(5.5)$ & $97(7.4)$ & 0.500 & $0.042 *$ \\
\hline $\begin{array}{l}\text { Fine grasp - movement - release - } \\
\mathrm{RH} \text { - value }\end{array}$ & $59.7(28.2)$ & $89.7(3)$ & $88.7(3.9)$ & $0.027 *$ & $0.028^{*}$ \\
\hline $\begin{array}{l}\text { Gross grasp - movement - release - } \\
\text { LH - value }\end{array}$ & $67.1(29.6)$ & $86.3(6)$ & $90(4.2)$ & 0.058 & $0.028^{*}$ \\
\hline $\begin{array}{l}\text { Fine grasp - movement - release - } \\
\text { LH - value }\end{array}$ & 72.4 & 88. & $88.5(2.9)$ & $0.027^{*}$ & $0.027 *$ \\
\hline Movement to left - RH - time & $92.4(5)$ & $95.3(6.7)$ & $96.3(2.3)$ & 0.173 & $0.046^{*}$ \\
\hline Movement to right - RH - time & $90.4(7.6)$ & $95.7(6.3)$ & $93.7(4.5)$ & $0.046^{*}$ & 0.225 \\
\hline Movement up - RH - time & $92.1(5)$ & $97.5(2.2)$ & $97(3.2)$ & $0.046^{*}$ & $0.027 *$ \\
\hline Movement down - RH - time & $88.2(9.7)$ & $94.2(4.5)$ & $95.2(2.5)$ & 0.080 & $0.043 *$ \\
\hline Movement backwards - RH - time & $94.3(4.7)$ & $96.5(3.6)$ & $98(2.4)$ & 0.131 & $0.027 *$ \\
\hline Movement to left - LH - time & $90.1(8.7)$ & $94.2(4.8)$ & $95.5(3.5)$ & $0.046^{*}$ & 0.080 \\
\hline Movement up - LH - time & $92.3(6.5)$ & $96.3(3.6)$ & $95.3(3.8)$ & $0.046^{*}$ & 0.144 \\
\hline $\begin{array}{l}\text { Gross grasp - movement - release - } \\
\text { RH - time }\end{array}$ & $61(27.3)$ & $88.7(3.7)$ & $90.7(2.9)$ & $0.028^{*}$ & $0.027 *$ \\
\hline $\begin{array}{l}\text { Fine grasp - movement - release - } \\
\text { RH - time }\end{array}$ & $59.7(28.7)$ & $89.7(3)$ & $88.7(3.9)$ & $0.027^{*}$ & $0.028 *$ \\
\hline $\begin{array}{l}\text { Gross grasp - movement - release - } \\
\text { LH - time }\end{array}$ & 67.1(29.6) & $86.3(6)$ & $90(4.2)$ & 0.058 & $0.028^{*}$ \\
\hline $\begin{array}{l}\text { Fine grasp - movement - release - } \\
\text { LH - time }\end{array}$ & $72.4(13.1)$ & $88.5(1.8)$ & $88.5(2.9)$ & $0.027 *$ & $0.027 *$ \\
\hline
\end{tabular}

$* \mathrm{p}<0.05$ 


\section{Discussion}

The aim of this study was to widen Occupational Therapist's standardized and validated instruments through the assessment of the efficacy of the SaeboReJoyce in the evaluation of changes in mobility and functionality of the upper limb in patients with PD.

Even if the evidence has not yet resulted in standardized guidelines, Virtual reality (VR) has recently been used as a tool for rehabilitation in individuals with different neurological disease, included PD. VR is able to facilitate motor learning for balance and gait rehabilitation resulting in additional benefits, especially when combined with other interventions such as conventional rehabilitation [16].

Thanks to the use of immersive VR, stress decreased, the level of arousal after exposure increased and there were not negative physiological and psychological effects [17].

Concerning the rating scale (PDQ-39, DASH, GDS and to the Jebsen test), a statistically significant difference not in all the items of the ReJoyce was found, however it is considered clinically relevant. For most items of the SaeboReJoyce a statistical difference was found with a $\mathrm{p}<0,05$.

\subsection{Limitations of the study}

Our study has two important limitations: the small sample size and the short duration of treatment.

\section{Conclusion}

Our data suggest that the SaeboReJoyce could be an useful instrument not only for the health professional dealing with PD but also for students for the evaluation of changes in functionality of the upper limb, including both gross and fine motor tasks/grasps, for patients with PD. It could be also useful for the health professional dealing with $\mathrm{PD}$, allowing them to elaborate a correct intervention strategy and modify it if necessary.

In order to better evaluate the effectiveness of SaeboReJoyce as standardized instrument for the evaluation of upper limb before, during and after rehabilitation, further studies.

\section{References}

1. De Lau, Lonneke ML, and Monique MB Breteler: "Epidemiology of Parkinson's disease." The Lancet Neurology 5(6), 525-535 (2006).

2. Valent F, Devigili G, Rinaldo S, Del Zotto S, Tullio A, Eleopra R. "The epidemiology of Parkinson's disease in the Italian region Friuli Venezia Giulia: a population-based study with administrative data." Neurological Sciences 39(4), 699-704 (2018). 
3. Dorsey ER, Bloem BR. "The Parkinson Pandemic - A Call to Action." JAMA Neurology 75(1), 9-10 (2018).

4. Alves G, Forsaa EB, Pedersen KF, Dreetz Gjerstad M, Larsen JP: "Epidemiology of Parkinson's disease." Journal of neurology 255(5), 18-32 (2008).

5. Clinical presentation: https://www.saebo.com/saebo-rejoyce/ last accessed 2019/01/11

6. Kowalczewski J, Chong S. L, Galea M, Prochazka A. "In-Home TeleRehabilitation Improves Tetraplegic Hand Function." Neurorehabilitation and Neural Repair 25, 412-422 (2011).

7. Kowalczewski J, Ravid E, Prochazka A, Fully-Automated Test of Upperextremity Function, 33rd Annual International Conference of the IEEE EMBS Boston, Massachusetts USA, August 30 - September 3, 2011;

8. Nasreddine ZS, Phillips NA, Bédirian V, Charbonneau S, Whitehead V, Colli I, Cummings JL, Chertkow H: "The Montreal Cognitive Assessment, MoCA: a brief screening tool for mild cognitive impairment." Journal of the American Geriatrics Society 53, 695-699 (2005).

9. Galeoto G, Colalelli F, Massai P, Berardi A, Tofani M, Pierantozzi M, Servadio A, Fabbrini A, Fabbrini G: "Quality of life in Parkinson's disease: Italian validation of the Parkinson's Disease Questionnaire (PDQ-39-IT)." Neurological Sciences 39, 1903-1909 (2018).

10. Padua R, Padua L, Ceccarelli E, Romanini E, Zanoli G, Amadio PC, Campi A: "Italian version of the disability of the arm, shoulder and hand (DASH) questionnaire. Cross-cultural adaptation and validation." Journal of Hand Surgery (British and European Volume) 28B(2), 179-186 (2003).

11. Culicchia G, Nobilia M, Asturi M, Santilli V, Paoloni M, De Santis R, Galeoto G: "Cross-Cultural Adaptation and Validation of the Jebsen-Taylor Hand Function Test in an Italian Population." Rehabilitation Research and Practice (2016).

12. Massai P, Colalelli F, Sansoni J, Valente D, Tofani M, Fabbrini G, Fabbrini A, Scuccimarri M, Galeoto G: "Reliability and Validity of the Geriatric Depression Scale in Italian Subjects with Parkinson's Disease.” Parkinson's Disease (2018).

13. Galeoto G, Sansoni J, Scuccimarri M, Bruni V, De Santis R, Colucci M, Valente D, Tofani M: "A Psychometric Properties Evaluation of the Italian Version of the Geriatric Depression Scale." Depression research and treatment (2018).

14. Galeoto G, De Santis R, Marcolini A, Cinelli A, Cecchi R. Il consenso informato in Terapia Occupazionale: proposta di una modulistica. G Ital Med Lav Ergon. 2016;38:107-15.

15. Galeoto G, Mollica R, Astorino O, Cecchi R. Informed consent in physiotherapy: proposal of a form. G Ital Med Lav Ergon. 37, 245-54 (2015).

16. Cano Porras D, Siesmonsma P, Inzelberg R, Zelig G, Plotnik M: "Advantages of virtual reality in the rehabilitation of balance and gait: Systematic review." Neurology 90, 1017-1025 (2018).

17. Kim A, Darakjian N, Finley JM. "Walking in fully immersive virtual environments: an evaluation of potential adverse effects in older adults and individuals with Parkinson's disease." Journal of NeuroEngineering and Rehabilitation 14, 16 (2017). 